Pacific

Journal of

Mathematics

\title{
ON A CLASS OF SEMIHEREDITARY
}

CROSSED-PRODUCT ORDERS

JOHN S. KAUTA

Volume $259 \quad$ No. 2

October 2012 


\title{
ON A CLASS OF SEMIHEREDITARY CROSSED-PRODUCT ORDERS
}

\author{
JOHN S. KAUTA
}

\begin{abstract}
Let $\boldsymbol{F}$ be a field, let $\boldsymbol{V}$ be a valuation ring of $\boldsymbol{F}$ of arbitrary Krull dimension (rank), let $K$ be a finite Galois extension of $F$ with group $G$, and let $S$ be the integral closure of $V$ in $K$. Let $f: G \times G \mapsto K \backslash\{0\}$ be a normalized twococycle such that $f(G \times G) \subseteq S \backslash\{0\}$, but we do not require that $f$ should take values in the group of multiplicative units of $S$. One can construct a crossed-product $V$-algebra $A_{f}=\sum_{\sigma \in G} S x_{\sigma}$ in a natural way, which is a $V$ order in the crossed-product $F$-algebra $(K / F, G, f)$. If $V$ is unramified and defectless in $K$, we show that $A_{f}$ is semihereditary if and only if for all $\sigma, \tau \in G$ and every maximal ideal $M$ of $S, f(\sigma, \tau) \notin M^{2}$. If in addition $J(V)$ is not a principal ideal of $V$, then $A_{f}$ is semihereditary if and only if it is an Azumaya algebra over $V$.
\end{abstract}

\section{Introduction}

In this paper we study certain orders over valuation rings in central simple algebras. If $R$ is a ring, then $J(R)$ will denote its Jacobson radical, $U(R)$ its group of multiplicative units, and $R^{\#}$ the subset of all the nonzero elements. The residue ring $R / J(R)$ will be denoted by $\bar{R}$. Given the ring $R$, it is called primary if $J(R)$ is a maximal ideal of $R$. It is called hereditary if one-sided ideals are projective $R$-modules. It is called semihereditary (respectively Bézout) if finitely generated one-sided ideals are projective $R$-modules (respectively are principal). Let $V$ be a valuation ring of a field $F$. If $Q$ is a finite-dimensional central simple $F$-algebra, then a subring $R$ of $Q$ is called an order in $Q$ if $R F=Q$. If in addition $V \subseteq R$ and $R$ is integral over $V$, then $R$ is called a $V$-order. If a $V$-order $R$ is maximal among the $V$-orders of $Q$ with respect to inclusion, then $R$ is called a maximal $V$-order (or just a maximal order if the context is clear). A $V$-order $R$ of $Q$ is called an extremal $V$-order (or simply extremal when the context is clear) if for every $V$-order $B$ in $Q$ with $B \supseteq R$ and $J(B) \supseteq J(R)$, we have $B=R$. If $R$ is an order in $Q$, then it is

MSC2010: 13F30, 16H05, 16E60, 16S35, 16H10.

Keywords: crossed-product orders, semihereditary orders, hereditary orders, Azumaya algebras,

Dubrovin valuation rings. 
called a Dubrovin valuation ring of $Q$ (or a valuation ring of $Q$ in short) if it is semihereditary and primary (see [Dubrovin 1982; 1984]).

In this paper, $V$ will denote a commutative valuation ring of arbitrary Krull dimension (rank). Let $F$ be its field of quotients, let $K / F$ be a finite Galois extension with group $G$, and let $S$ be the integral closure of $V$ in $K$. If $f \in Z^{2}(G, U(K))$ is a normalized two-cocycle such that $f(G \times G) \subseteq S^{\#}$, then one can construct a "crossed-product" $V$-algebra

$$
A_{f}=\sum_{\sigma \in G} S x_{\sigma}
$$

with the usual rules of multiplication $\left(x_{\sigma} s=\sigma(s) x_{\sigma}\right.$ for all $s \in S, \sigma \in G$ and $x_{\sigma} x_{\tau}=$ $\left.f(\sigma, \tau) x_{\sigma \tau}\right)$. Then $A_{f}$ is associative, with identity $1=x_{1}$, and center $V=V x_{1}$. Further, $A_{f}$ is a $V$-order in the crossed-product $F$-algebra $\Sigma_{f}=\sum_{\sigma \in G} K x_{\sigma}=$ $(K / F, G, f)$. Following [Haile 1987], we let $H=\left\{\sigma \in G \mid f\left(\sigma, \sigma^{-1}\right) \in U(S)\right\}$. Then $H$ is a subgroup of $G$.

In this paper, we will always assume that $V$ is unramified and defectless in $K$ (for the definitions of these terms, see [Endler 1972]). By [Endler 1972, Theorem 18.6], $S$ is a finitely generated $V$-module, hence $A_{f}$ is always finitely generated over $V$. If $V_{1}$ is a valuation ring of $K$ lying over $V$ then $\left\{\sigma \in G \mid \sigma(x)-x \in J\left(V_{1}\right) \forall x \in V_{1}\right\}$ is called the inertial group of $V_{1}$ over $F$. By [Kauta 2001, Lemma 1], the condition that $V$ is unramified and defectless in $K$ is equivalent to saying that the inertial group of $V_{1}$ over $F$ is trivial, since $K / F$ is a finite Galois extension.

These orders were first studied in [Haile 1987], and later in [Haile and Morandi 1993; Kauta 2012]. In [Haile 1987; Kauta 2012], only the case when $V$ is a discrete valuation ring (DVR) was considered. In [Kauta 2012], hereditary properties of crossed-product orders were examined. In [Haile 1987; Haile and Morandi 1993], valuation ring properties of the crossed-product orders were explored, and the latter considered the cases when either $V$ has arbitrary Krull dimension but is indecomposed in $K$, or $V$ is a discrete finite-rank valuation ring, that is, its value group is $\mathbb{Z} \oplus \cdots \oplus \mathbb{Z}$. When $V$ is a DVR, then any $V$-order in $\Sigma_{f}$ containing $S$ is a crossed-product order of the form $A_{g}$ for some two-cocycle $g: G \times G \mapsto S^{\#}$, with $g$ cohomologous to $f$ over $K$, by [Haile 1987, Proposition 1.3], but this need not be the case in general. While [Haile and Morandi 1993] considered any $V$-order in $\Sigma_{f}$ containing $S$, some of which were not of the type described above and so in that sense its scope was wider than ours, in this paper we shall only be concerned with crossed-product orders $A_{g}$ where $g$ is either $f$ (almost always), or is cohomologous to $f$ over $K$, that is, if there are elements $\left\{c_{\sigma} \mid \sigma \in G\right\} \subseteq K^{\#}$ such that $g(\sigma, \tau)=c_{\sigma} \sigma\left(c_{\tau}\right) c_{\sigma \tau}^{-1} f(\sigma, \tau)$ for all $\sigma, \tau \in G$, a fact denoted by $g \sim_{K} f$.

The purpose of this paper is to generalize the results of [Kauta 2012] to the case when $V$ is not necessarily a DVR. The main results of this paper are as follows: 
$A_{f}$ is semihereditary if and only if for all $\sigma, \tau \in G$ and every maximal ideal $M$ of $S, f(\sigma, \tau) \notin M^{2}$; if $J(V)$ is not a principal ideal of $V$, then $A_{f}$ is semihereditary if and only if it is an Azumaya algebra over $V$. As in [Kauta 2012], the utility of these criteria lie in their simplicity.

Although in our case the valuation ring $V$ need not be a DVR, some of the steps in the proofs in [Haile 1987; Kauta 2012] remain valid, mutatis mutandis, owing to the theory developed in [Kauta 1997a; 1997b]. We shall take full advantage of this whenever the opportunity arises. Aside from the difficulties inherent when dealing with $V$-orders that are not necessarily noetherian, the hurdles encountered in this theory arise mainly due to the fact that the two-cocycle $f$ is not assumed to take on values in $U(S)$.

\section{Preliminaries}

In this section, we gather together various results that will help us prove the main results of this paper, which are in the next section. For the convenience of the reader, we have included complete proofs whenever it warrants, although the arguments are sometimes routine.

The following lemma is essentially embedded in the proof of [Kauta 1997a, Proposition 1.8], and the remark that follows it.

Lemma 2.1. Let $A$ be a finitely generated extremal $V$-order in a finite-dimensional central simple $F$-algebra $Q$.

(1) If $B$ is a $V$-order of $Q$ containing $A$, then $B$ is also a finitely generated extremal order. If in addition $B$ is a maximal $V$-order, then it is a valuation ring of $Q$.

(2) If $W$ is an overring of $V$ in $F$ with $V \varsubsetneqq W$, then $W A$ is a valuation ring of $Q$ with center $W$.

Proof. Let $B$ be a $V$-order containing $A$. By [Kauta 1997a, Proposition 1.8], $A$ is semihereditary, hence $B$ is semihereditary by [Morandi 1992, Lemma 4.10], and therefore $B$ is extremal by [Kauta 1997a, Theorem 1.5]. Since $[B / J(B)$ : $V / J(V)] \leq\left[\Sigma_{f}: F\right]<\infty$, there exists $a_{1}, a_{2}, \ldots, a_{m} \in B$ such that $B=a_{1} V+$ $a_{2} V+\cdots+a_{m} V+J(B)$. But by [Kauta 1997a, Proposition 1.4], $J(B) \subseteq J(A)$, since $A$ is extremal. Therefore $B=a_{1} V+a_{2} V+\cdots+a_{m} V+A$, a finitely generated $V$-order. If, in addition, $B$ is a maximal $V$-order, then by the remark after [Kauta 1997a, Proposition 1.8], $B$ is a valuation ring of $Q$.

Now let $W$ be a proper overring of $V$ in $F$. Let $C$ be a maximal $V$-order containing $A$. Then $C$ is a valuation ring of $Q$, as seen above, hence $W C$ is a valuation ring of $Q$ with center $W$. Since $A$ is an extremal $V$-order, we have $J(C) \subseteq J(A)$, thus $W C=W J(V) C \subseteq W J(C) \subseteq W A \subseteq W C$, so that $W A=W C$. Thus $W A$ is always a valuation ring of $Q$. 
Since $A_{f}$ is finitely generated over $V$, we immediately have the following lemma, because of [Kauta 1997a, Proposition 1.8], the remark that follows it, and the fact that Bézout $V$-orders are maximal orders by [Morandi 1992, Theorem 3.4].

Lemma 2.2. Given the crossed-product order $A_{f}$,

(1) it is an extremal order if and only if it is semihereditary and

(2) it is a maximal order if and only if it is a valuation ring, if and only if it is Bézout.

Lemma 2.3. Let $W$ be a valuation ring of $F$ such that $V \varsubsetneqq W$, and let $R=W S$.

(1) Then $R$ is the integral closure of $W$ in $K$, and $W$ is also unramified and defectless in $K$.

(2) Let $t \in S$ satisfy $t \notin M^{2}$ for every maximal ideal $M$ of $S$. Then $t \in U(R)$. If in addition $J(V)$ is a nonprincipal ideal of $V$, then $t \in U(S)$.

Proof. The ring $R$ is obviously integral over $W$. Since it contains $S$, it is also integrally closed in $K$, hence it is the integral closure of $W$ in $K$.

Now let $V_{1} \subseteq W_{1}$ be valuation rings of $K$ lying over $V$ and $W$ respectively. Then $J\left(W_{1}\right) \subseteq J\left(V_{1}\right)$; hence the inertial group of $W_{1}$ over $F$, namely

$$
\left\{\sigma \in G \mid \sigma(x)-x \in J\left(W_{1}\right) \forall x \in W_{1}\right\},
$$

is contained in the inertial group of $V_{1}$ over $F,\left\{\sigma \in G \mid \sigma(x)-x \in J\left(V_{1}\right) \forall x \in V_{1}\right\}$. Since $V$ is unramified and defectless in $K$, the latter group is trivial, forcing $W$ to be unramified and defectless in $K$.

Let $W_{1}$ be a valuation ring of $K$ lying over $W$, and let $V_{1}$ be a valuation ring of $K$ lying over $V$ such that $V_{1} \subseteq W_{1}$, as in the preceding paragraph. Let $M=J\left(V_{1}\right) \cap S$, a generic maximal ideal of $S$. We claim that $M^{2}=J\left(V_{1}\right)^{2} \cap S$. To see this, note that

$$
M^{2}=\left(J\left(V_{1}\right) \cap S\right)\left(J\left(V_{1}\right) \cap S\right) \subseteq J\left(V_{1}\right)^{2} \cap S
$$

and

$$
M^{2} V_{1}=\left(J\left(V_{1}\right) \cap S\right)\left(J\left(V_{1}\right) \cap S\right) V_{1}=J\left(V_{1}\right)^{2}=\left(J\left(V_{1}\right)^{2} \cap S\right) V_{1} .
$$

If $V^{\prime}$ is an extension of $V$ to $K$ different from $V_{1}$, then $M^{2} V^{\prime}=V^{\prime}=\left(J\left(V_{1}\right)^{2} \cap S\right) V^{\prime}$. Thus $M^{2}=J\left(V_{1}\right)^{2} \cap S$ as desired. If $t \in S$ satisfies $t \notin M^{2}$, then $t \notin J\left(V_{1}\right)^{2}$. Since $J\left(W_{1}\right) \varsubsetneqq J\left(V_{1}\right)^{2}$, we have $t \in U\left(W_{1}\right)$. Since $W_{1}$ was an arbitrary extension of $W$ in $K$, we conclude that $t \in U(R)$. If $J(V)$ is a nonprincipal ideal of $V$, then $J\left(V_{1}\right)^{2}=J\left(V_{1}\right)$, hence $t \in U\left(V_{1}\right)$ for every such extension $V_{1}$ of $V$ to $K$, and we conclude that $t \in U(S)$.

Part (4) of the following lemma was originally proved in [Haile 1987] when $V$ is a DVR. The same arguments work when $V$ is an arbitrary valuation ring. 
Lemma 2.4. Given a $\sigma \in G$, let $I_{\sigma}=\bigcap M$, where the intersection is taken over those maximal ideals $M$ of $S$ for which $f\left(\sigma, \sigma^{-1}\right) \notin M$. Then:

(1) $I_{\sigma}=\left\{x \in S \mid x f\left(\sigma, \sigma^{-1}\right) \in J(V) S\right\}$.

(2) $I_{\sigma}^{\sigma^{-1}}=I_{\sigma^{-1}}$.

(3) If $f\left(\sigma, \sigma^{-1}\right) \notin M^{2}$ for every maximal ideal $M$ of $S$, then $I_{\sigma} f\left(\sigma, \sigma^{-1}\right)=$ $J(V) S$.

(4) $J\left(A_{f}\right)=\sum_{\sigma \in G} I_{\sigma} x_{\sigma}$.

Proof. Let $x \in S$. Clearly, if $x \in I_{\sigma}$ then $x f\left(\sigma, \sigma^{-1}\right) \in J(V) S$. On the other hand, if $x \notin I_{\sigma}$ then there exists a maximal ideal $M$ of $S$ such that $x, f\left(\sigma, \sigma^{-1}\right) \notin M$, hence $x f\left(\sigma, \sigma^{-1}\right) \notin M$, and thus $x f\left(\sigma, \sigma^{-1}\right) \notin J(V) S$.

The second statement is proved in the same manner as [Kauta 2012, Sublemma]. To see that the third statement holds, we note that $I_{\sigma} f\left(\sigma, \sigma^{-1}\right) \subseteq J(V) S$. We claim that $I_{\sigma} f\left(\sigma, \sigma^{-1}\right)=J(V) S$. To see this, let $M$ be a maximal ideal of $S$. If $f\left(\sigma, \sigma^{-1}\right) \notin M$, then $\left(I_{\sigma} f\left(\sigma, \sigma^{-1}\right)\right) S_{M}=J\left(S_{M}\right)=(J(V) S) S_{M}$. On the other hand, if $f\left(\sigma, \sigma^{-1}\right) \in M$ then, since $f\left(\sigma, \sigma^{-1}\right) \notin M^{2}$, we have $J\left(S_{M}\right)^{2} \varsubsetneqq$ $I_{\sigma} f\left(\sigma, \sigma^{-1}\right) S_{M} \subseteq J\left(S_{M}\right)$, hence $I_{\sigma} f\left(\sigma, \sigma^{-1}\right) S_{M}=J\left(S_{M}\right)=(J(V) S) S_{M}$, and thus $I_{\sigma} f\left(\sigma, \sigma^{-1}\right)=J(V) S$. By [Haile and Morandi 1993, Lemma 1.3], $J\left(A_{f}\right)=$ $\sum_{\sigma \in G}\left(J\left(A_{f}\right) \cap S x_{\sigma}\right)$. Therefore the fourth statement can be verified in exactly the same manner as [Haile 1987, Proposition 3.1(b)], because of the observations made above.

The following lemma is a generalization of [Haile 1987, Proposition 1.3].

Lemma 2.5. Let $B \subseteq \Sigma_{f}$ be a V-order. There is a normalized cocycle $g: G \times$ $G \mapsto S^{\#}, g \sim_{K} f$, such that $B=A_{g}$ (viewed as a subalgebra of $\Sigma_{f}$ in a natural way) if and only if $B \supseteq S$ and $B$ is finitely generated over $V$. When this occurs, $B=\sum_{\sigma \in G} S k_{\sigma} x_{\sigma}$ for some $k_{\sigma} \in K^{\#}$.

Proof. Suppose $B \supseteq S$. By [Haile and Morandi 1993, Lemma 1.3], $B=\sum_{\sigma \in G} B_{\sigma} x_{\sigma}$, where each $B_{\sigma}$ is a nonzero $S$-submodule of $K$. If in addition $B$ is finitely generated over $V$, then each $B_{\sigma}$ is finitely generated over $V$ : if $B=\sum_{i=1}^{n} V y_{i}$ then, if we write $y_{i}=\sum_{\tau \in G} k_{\tau}^{(i)} x_{\tau}$ with $k_{\tau}^{(i)} \in K$, we see that $B_{\sigma}$ is generated by $\left\{k_{\sigma}^{(i)}\right\}_{i=1}^{n}$ over $V$. Since $S$ is a commutative Bézout domain with $K$ as its field of quotients, $B_{\sigma}=S k_{\sigma}$ for some $k_{\sigma} \in K^{\#}$. Thus we get $B=\sum_{\sigma \in G} S k_{\sigma} x_{\sigma}$. Since $B$ is integral over $V, B_{1}=S$ and so we can choose $k_{1}=1$. Define $g: G \times G \mapsto S^{\#}$ by $g(\sigma, \tau) k_{\sigma \tau} x_{\sigma \tau}=\left(k_{\sigma} x_{\sigma}\right)\left(k_{\tau} x_{\tau}\right)$, as in [Haile 1987, Proposition 1.3]. Since $k_{1}=1$, $g$ is also a normalized two-cocycle. The converse is obvious.

Lemma 2.6. Suppose $S$ is a valuation ring of $K$. Then

$J(V) A_{f}$ is a maximal ideal of $A_{f} \Longleftrightarrow H=G \quad \Longleftrightarrow \quad A_{f}$ is Azumaya over $V$. 
Proof. Suppose $J(V) A_{f}$ is a maximal ideal of $A_{f}$. Note that $A_{f} / J(V) A_{f}=$ $\sum_{\sigma \in G} \bar{S} \tilde{x}_{\sigma}$. By [Haile et al. 1983, Theorem 10.1(c)], $J=\sum_{\sigma \notin H} \bar{S} \tilde{x}_{\sigma}$ is an ideal of $A_{f} / J(V) A_{f}$. Since $A_{f} / J(V) A_{f}$ is simple, $J=0$, hence $H=G$.

We set up additional notation, following [Haile 1987; Kauta 2012]. Let $L$ be an intermediate field of $F$ and $K$, let $G_{L}$ be the Galois group of $K$ over $L$, let $U$ be a valuation ring of $L$ lying over $V$, and let $T$ be the integral closure of $U$ in $K$. Then one can obtain a two-cocycle $f_{L, U}: G_{L} \times G_{L} \mapsto T^{\#}$ from $f$ by restricting $f$ to $G_{L} \times G_{L}$, and embedding $S^{\#}$ in $T^{\#}$. As before, $A_{f_{L, U}}=\sum_{\sigma \in G_{L}} T x_{\sigma}$ is a $U$-order in $\Sigma_{f_{L, U}}=\sum_{\sigma \in G_{L}} K x_{\sigma}=\left(K / L, G_{L}, f_{L, U}\right)$, and $U$ is unramified and defectless in $K$. If $M$ is a maximal ideal of $S$, and $L$ is the decomposition field of $M$ and $U=L \cap S_{M}$, then we will denote $f_{L, U}$ by $f_{M}, A_{f_{L, U}}$ by $A_{f_{M}}, \Sigma_{f_{L, U}}$ by $\Sigma_{f_{M}}, L$ by $K_{M}$, and the decomposition group $G_{L}$ by $D_{M}$, as in [Haile 1987]. Further, we let $H_{M}=\left\{\sigma \in D_{M} \mid f_{M}\left(\sigma, \sigma^{-1}\right) \in U\left(S_{M}\right)\right\}$, a subgroup of $D_{M}$.

Given a maximal ideal $M$ of $S$, let $M=M_{1}, M_{2}, \ldots, M_{r}$ be the complete list of maximal ideals of $S$, let $U_{i}=S_{M_{i}} \cap K_{M_{i}}$ with $U=U_{1}$, and let $\left(K_{i}, S_{i}\right)$ be a Henselization of $\left(K, S_{M_{i}}\right)$. Let $\left(F_{h}, V_{h}\right)$ be the unique Henselization of $(F, V)$ contained in $\left(K_{1}, S_{1}\right)$. We note that $\left(F_{h}, V_{h}\right)$ is also a Henselization of $\left(K_{M}, U\right)$. By [Haile et al. 1995, Proposition 11], we have $S \otimes_{V} V_{h} \cong S_{1} \oplus S_{2} \oplus \cdots \oplus S_{r}$.

Part (1) of the following lemma was originally proved in [Haile 1987] in the case when $V$ is a DVR. Virtually the same proof holds in the general case. Part (2c) is a generalization of [Haile 1987, Corollary 3.11].

Lemma 2.7. Let the notation be as above.

(1) The crossed-product order $A_{f}$ is primary if and only if for every maximal ideal $M$ of $S$ there is a set of right coset representatives $g_{1}, g_{2}, \ldots, g_{r}$ of $D_{M}$ in $G$ (that is, $G$ is the disjoint union $\left.\bigcup_{i} D_{M} g_{i}\right)$ such that for all $i, f\left(g_{i}, g_{i}^{-1}\right) \notin M$.

(2) Assume the crossed-product order $A_{f}$ is primary. Then:

(a) $A_{f} \otimes_{V} V_{h} \cong M_{r}\left(A_{f_{M}} \otimes_{U} V_{h}\right)$.

(b) As a result of (a), $A_{f} / J\left(A_{f}\right) \cong M_{r}\left(A_{f_{M}} / J\left(A_{f_{M}}\right)\right)$.

(c) Also as a result of (a), $A_{f}$ is a valuation ring of $\Sigma_{f}$ if and only if $A_{f_{M}}$ is a valuation ring of $\Sigma_{f_{M}}$ for some maximal ideal $M$ of $S$. When this occurs, $A_{f_{M}}$ is a valuation ring of $\Sigma_{f_{M}}$ for every maximal ideal $M$ of $S$.

(d) $A_{f}$ is Azumaya over $V$ if and only if $H_{M}=D_{M}$ for some maximal ideal $M$ of $S$. When this occurs, $H_{M}=D_{M}$ for every maximal ideal $M$ of $S$.

Proof. The proof of [Haile 1987, Theorem 3.2], appropriately adapted, works here as well to establish part (1). We outline the argument, for the convenience of the reader: For a $\sigma \in G$, let $I_{\sigma}$ be as in Lemma 2.4, and, for a maximal ideal $M$ of $S$, set $\hat{M}:=\bigcap_{N \text { max, } N \neq M} N$. If $I$ is an ideal of $A_{f}$ then, by [Haile and Morandi 1993, Lemma 1.3], $I=\sum_{\sigma \in G}\left(I \cap S x_{\sigma}\right)$, so $A_{f}$ is primary if and only if 
the following condition holds: if $\sigma \in G$ and $T$ is an ideal of $S$ such that $T \nsubseteq I_{\sigma}$, then $A_{f} T x_{\sigma} A_{f}=A_{f}$.

If $A_{f}$ is primary and $M$ is a maximal ideal of $S$, then $A_{f}=A_{f} \hat{M} x_{1} A_{f}$. Therefore if $G=\bigcup_{j=1}^{r} h_{j} D_{M}$ is a left coset decomposition, then

$$
S=\sum_{j} \hat{M}^{h_{j}}\left(\sum_{d \in D_{M}} f\left(h_{j} d, d^{-1} h_{j}^{-1}\right)\right),
$$

as in the proof of [Haile 1987, Theorem 3.2], so that, if we fix $i, 1 \leq i \leq r$, and localize at $M^{h_{i}}$, we get

$$
S_{M^{h_{i}}}=\sum_{j \neq i} J\left(S_{M^{h_{i}}}\right)\left(\sum_{d \in D_{M}} f\left(h_{j} d, d^{-1} h_{j}^{-1}\right)\right)+S_{M^{h_{i}}}\left(\sum_{d \in D_{M}} f\left(h_{i} d, d^{-1} h_{i}^{-1}\right)\right),
$$

and hence $\sum_{d \in D_{M}} f\left(h_{i} d, d^{-1} h_{i}^{-1}\right) \notin M^{h_{i}}$. So there is an element $d_{i} \in D_{M}$ such that $f\left(h_{i} d_{i}, d_{i}^{-1} h_{i}^{-1}\right) \notin M^{h_{i}}$. Let $g_{i}=d_{i}^{-1} h_{i}^{-1}$. Then $g_{1}, g_{2}, \ldots, g_{r}$ have the desired properties.

For the converse, suppose $\sigma \in G$ and $T$ is an ideal of $S$ such that $T \nsubseteq I_{\sigma}$. We need to show that $A_{f} T x_{\sigma} A_{f}=A_{f}$. Since $T \nsubseteq I_{\sigma}$, there is a maximal ideal $M$ of $S$ such that $f\left(\sigma, \sigma^{-1}\right) \notin M$ and $T \nsubseteq M$. The argument in [Haile 1987, Theorem 3.2] shows that $A_{f} T x_{\sigma} A_{f} \supseteq \sum_{i=1}^{r} T_{i}$, where $T_{i}=T^{g_{i}^{-1}} f^{g_{i}^{-1}}\left(\sigma, \sigma^{-1} g_{i}\right) f\left(g_{i}^{-1}, g_{i}\right)$ are ideals of $S$ satisfying the condition $T_{i} \nsubseteq M^{g_{i}^{-1}}$. Inasmuch as $g_{1}^{-1}, g_{2}^{-1}, \ldots, g_{r}^{-1}$ form a complete set of left coset representatives of $D_{M}$ in $G$, the ideal $\sum_{i=1}^{r} T_{i}$ is not contained in any maximal ideal of $S$. Therefore $\sum_{i=1}^{r} T_{i}=S$, and so $A_{f} T x_{\sigma} A_{f}=A_{f}$.

Using part (1) and the fact that $S \otimes_{V} V_{h} \cong S_{1} \oplus S_{2} \oplus \cdots \oplus S_{r}$, we can construct a full set of matrix units in $A_{f} \otimes_{V} V_{h}$ and hence verify part (2a), as in the proof of [Haile 1987, Theorem 3.12] (see also the remark following that theorem). Part (2b) follows from (2a) and [Kauta 1997a, Lemma 3.1]; part (2c) follows from (2a); and (2d) follows from (2a) and Lemma 2.6.

\section{The main results}

We now give the main results of this paper. There are essentially two parallel theories: one takes effect when $J(V)$ is a principal ideal of $V$, and the other when it is not. In the former case, the order $A_{f}$ displays characteristics akin to the situation when $V$ is a DVR. Our theory, however, yields surprising results in the latter case. It turns out in this case that the property that $A_{f}$ is Azumaya over $V$ is equivalent to a much weaker property: that it is an extremal $V$-order in $\Sigma_{f}$.

Proposition 3.1. The order $A_{f}$ is Azumaya over $V$ if and only if $H=G$.

Proof. Suppose $A_{f}$ is Azumaya over $V$. Let $M$ be a maximal ideal of $S$. By Lemma 2.7(1), there is a set of right coset representatives $g_{1}, g_{2}, \ldots, g_{r}$ of $D_{M}$ 
in $G$ such that $f\left(g_{i}, g_{i}^{-1}\right) \notin M$. If $\sigma \in G$, then $\sigma=h g_{i}$ for some $h \in D_{M}$ and some $i$. Since $A_{f}$ is Azumaya, $H_{M}=D_{M}$ by Lemma 2.7(2d), hence we have $f\left(h^{-1}, h\right) \notin M$. Because

$$
f^{h^{-1}}\left(h g_{i}, g_{i}^{-1} h^{-1}\right) f^{h^{-1}}\left(h, g_{i}\right) f^{g_{i}}\left(g_{i}^{-1}, h^{-1}\right)=f\left(h^{-1}, h\right) f\left(g_{i}, g_{i}^{-1}\right),
$$

we conclude that $f\left(\sigma, \sigma^{-1}\right) \notin M$. Since $M$ is arbitrary, $f\left(\sigma, \sigma^{-1}\right) \in U(S)$ for every $\sigma \in G$, so that $H=G$.

The converse is well-known and straightforward to demonstrate.

It is perhaps instructive to compare the above proposition to [Kauta 2001, Theorem 3].

Recall that $J(V)$ is a nonprincipal ideal of $V$ if and only if $J(V)^{2}=J(V)$.

Proposition 3.2. Suppose $J(V)$ is a nonprincipal ideal of $V$. Then the following statements about the crossed-product order $A_{f}$ are equivalent:

(1) $A_{f}$ is an extremal $V$-order in $\Sigma_{f}$.

(2) $A_{f}$ is a semihereditary $V$-order.

(3) $A_{f}$ is a maximal $V$-order in $\Sigma_{f}$.

(4) $A_{f}$ is a Bézout V-order.

(5) $A_{f}$ is a valuation ring of $\Sigma_{f}$.

(6) Af is Azumaya over $V$.

Proof. By Lemma 2.2, it suffices to demonstrate that (1) $\Rightarrow(5) \Rightarrow(6)$. So suppose $A_{f}$ is an extremal $V$-order. Let $B$ be a maximal $V$-order containing $A_{f}$. By Lemma 2.1, $B$ is a valuation ring finitely generated over $V$. By Lemma 2.5 , we get that $B=\sum_{\sigma \in G} S k_{\sigma} x_{\sigma}$ for some $k_{\sigma} \in K^{\#}$. Since $A_{f}$ is extremal, we have $J(B) \subseteq J\left(A_{f}\right)$ by [Kauta 1997a, Proposition 1.4], so $J(V) B \subseteq A_{f}$. Therefore $\sum_{\sigma \in G} J(S) k_{\sigma} x_{\sigma}=$ $J(V) B=J(V)^{2} B \subseteq J(V) A_{f}=\sum_{\sigma \in G} J(S) x_{\sigma}$, so that $J(S) k_{\sigma} \subseteq J(S)$. Hence for each maximal ideal $M$ of $S$, we have $S_{M} J(S) k_{\sigma} \subseteq S_{M} J(S)$, that is, $J\left(S_{M}\right) k_{\sigma} \subseteq$ $J\left(S_{M}\right)$. This shows that $k_{\sigma} \in S_{M}$ for all $M$ and so $k_{\sigma} \in S$ for every $\sigma \in G$, and thus $A_{f}=B$, a valuation ring.

Now suppose $A_{f}$ is a valuation ring of $\Sigma_{f}$. By Lemma 2.7(2), to show that $A_{f}$ is Azumaya over $V$, we may as well assume $S$ is a valuation ring of $K$. By [Dubrovin 1984, §2, Theorem 1], $J\left(A_{f}\right)=J(V) A_{f}$, and so $A_{f}$ is Azumaya over $V$ by Lemma 2.6 .

Remark. It follows from Lemma 2.3(2) and Proposition 3.1 that, if $J(V)$ is a nonprincipal ideal of $V$, then the crossed-product order $A_{f}$ is extremal if and only if for all $\tau, \gamma \in G$ and every maximal ideal $M$ of $S, f(\tau, \gamma) \notin M^{2}$. 
If $W$ is a valuation ring of $F$ such that $V \varsubsetneqq W$, then we will denote by $B_{f}$ the $W$-order $W A_{f}=\sum_{\sigma \in G} R x_{\sigma}$, where $R=W S$ is the integral closure of $W$ in $K$ by Lemma 2.3. Recall that $W$ is also unramified and defectless in $K$.

Proposition 3.3. Suppose $J(V)$ is a principal ideal of $V$. Then $A_{f}$ is semihereditary if and only if for all $\tau, \gamma \in G$ and every maximal ideal $M$ of $S, f(\tau, \gamma) \notin M^{2}$.

Proof. The result holds when the Krull dimension of $V$ is one, by [Kauta 2012, Corollary], since $V$ is a DVR in this case. So let us assume from now on that the Krull dimension of $V$ is greater than one.

Let $p=\bigcap_{n \geq 1} J(V)^{n}$. Then $p$ is a prime ideal of $V, W=V_{p}$ is a minimal overring of $V$ in $F$, and $\tilde{V}=V / J(W)$ is a DVR of $\bar{W}$. Set $B_{f}=W A_{f}$, as above.

Suppose $A_{f}$ is semihereditary. We will show that for each $\tau \in G$ and each maximal ideal $M$ of $S, f\left(\tau, \tau^{-1}\right) \notin M^{2}$.

First, assume that $V$ is indecomposed in $K$. By [Haile and Morandi 1993, Proposition 2.6], $A_{f}$ is primary, hence it is a valuation ring of $\Sigma_{f}$. Therefore $B_{f}$ is Azumaya over $W$, by [Haile and Morandi 1993, Proposition 2.10], and $f(G \times G) \subseteq U(R)$, by Proposition 3.1. Observe that $R$ is a valuation ring of $K$ lying over $W$ and $\bar{R}$ is Galois over $\bar{W}$, with group $G$, and $B_{f} / J\left(B_{f}\right)=\sum_{\sigma \in G} \bar{R} \tilde{x}_{\sigma}$ is a crossed-product $\bar{W}$-algebra. Further, $A_{f} / J\left(B_{f}\right)$ has center $\tilde{V}$, a DVR of $\bar{W}$, and is a crossed-product $\tilde{V}$-order in $B_{f} / J\left(B_{f}\right)$ of the type under consideration in this paper, since $\tilde{V}$ is unramified in $\bar{R}$ and $f(G \times G) \subseteq S \cap U(R)$. Since the crossedproduct $\tilde{V}$-order $A_{f} / J\left(B_{f}\right)$ is a valuation ring of $B_{f} / J\left(B_{f}\right)$ and hence hereditary, it follows from [Kauta 2012, Theorem] that for each $\tau \in G, f\left(\tau, \tau^{-1}\right) \notin J(S)^{2}$.

Suppose $V$ is not necessarily indecomposed in $K$, but assume $A_{f}$ is a valuation ring. Fix a maximal ideal $M$ of $S$. By Lemma 2.7(1), there is a set of right coset representatives $g_{1}, g_{2}, \ldots, g_{r}$ of $D_{M}$ in $G$ such that $f\left(g_{i}, g_{i}^{-1}\right) \notin M$. If $\tau \in G$, then $\tau=h g_{i}$ for some $h \in D_{M}$ and some $i$. By Lemma 2.7(2), $A_{f_{M}}$ is a valuation ring of $\Sigma_{f_{M}}$. Hence, by the preceding paragraph, $f_{M}\left(h^{-1}, h\right) \notin M^{2}$, and thus $f\left(h^{-1}, h\right) \notin M^{2}$. But the following holds:

$$
f^{h^{-1}}\left(h g_{i}, g_{i}^{-1} h^{-1}\right) f^{h^{-1}}\left(h, g_{i}\right) f^{g_{i}}\left(g_{i}^{-1}, h^{-1}\right)=f\left(h^{-1}, h\right) f\left(g_{i}, g_{i}^{-1}\right) .
$$

Therefore we must have $f\left(\tau, \tau^{-1}\right) \notin M^{2}$.

Now suppose that $A_{f}$ is not necessarily a valuation ring. To show that for each $\tau \in G$ and each maximal ideal $M$ of $S$ we have $f\left(\tau, \tau^{-1}\right) \notin M^{2}$, one only needs to emulate the corresponding steps in the proof of [Kauta 2012, Theorem], equipped with the following four observations:

1. Any maximal $V$-order containing $A_{f}$ is a valuation ring, by Lemma 2.1 , hence $A_{f}$ is the intersection of finitely many valuation rings all with center $V$, since $J(V)$ is a principal ideal of $V$, by [Kauta 1997b, Theorem 2.5]. 
2. If $B$ is one such valuation ring containing $A_{f}$, then $B=A_{g}=\sum_{\tau \in G} S k_{\tau} x_{\tau}$ for some $k_{\tau} \in K^{\#}$, where $g: G \times G \mapsto S^{\#}$ is some normalized two-cocycle, by Lemma 2.1(1) and Lemma 2.5. Fix $\sigma \in G$ and a maximal ideal $N$ of $S$. We may choose $B$ such that $k_{\sigma} \in U\left(S_{N}\right)$, as in the proof of [Kauta 2012, Theorem].

3. Both $J\left(A_{f}\right)$ and $J\left(A_{g}\right)$ are as in Lemma 2.4, that is, $J\left(A_{f}\right)=\sum_{\sigma \in G} I_{\sigma} x_{\sigma}$ (respectively $\left.J\left(B_{f}\right)=\sum_{\sigma \in G} J_{\sigma} k_{\sigma} x_{\sigma}\right)$ where $I_{\sigma}=\bigcap M$ (respectively $J_{\sigma}=\bigcap M$ ), as $M$ runs through all maximal ideals of $S$ for which $f\left(\sigma, \sigma^{-1}\right) \notin M$ (respectively $\left.g\left(\sigma, \sigma^{-1}\right) \notin M\right)$. We have $J\left(A_{g}\right) \subseteq J\left(A_{f}\right)$ by [Kauta 1997a, Theorem 1.5].

4. By Lemma 2.4, $I_{\sigma}^{\sigma^{-1}}=I_{\sigma^{-1}}, J_{\sigma}^{\sigma^{-1}}=J_{\sigma^{-1}}$, and $J_{\sigma^{-1}} g\left(\sigma^{-1}, \sigma\right)=J(V) S$.

We conclude, as in the proof of [Kauta 2012, Theorem], that

$$
J(V) S \subseteq k_{\sigma} I_{\sigma} f\left(\sigma, \sigma^{-1}\right) .
$$

Since $k_{\sigma} \in U\left(S_{N}\right)$, if $f\left(\sigma, \sigma^{-1}\right) \in N^{2}$ then, localizing both sides of (1) at $N$ we get $J\left(S_{N}\right) \subseteq J\left(S_{N}\right)^{2}$, a contradiction, since $J(V)$ is a principal ideal of $V$. Therefore for each $\tau \in G$ and each maximal ideal $M$ of $S, f\left(\tau, \tau^{-1}\right) \notin M^{2}$. Since the cocycle identity $f^{\tau}\left(\tau^{-1}, \tau \gamma\right) f(\tau, \gamma)=f\left(\tau, \tau^{-1}\right)$ holds, we conclude that for all $\tau, \gamma \in G$ and every maximal ideal $M$ of $S, f(\tau, \gamma) \notin M^{2}$.

Conversely, suppose $f(\tau, \gamma) \notin M^{2}$ for all $\tau, \gamma \in G$, and every maximal ideal $M$ of $S$. Let $O_{l}\left(J\left(A_{f}\right)\right)=\left\{x \in \Sigma_{f} \mid x J\left(A_{f}\right) \subseteq J\left(A_{f}\right)\right\}$. We will first establish that $O_{l}\left(J\left(A_{f}\right)\right)=A_{f}$, again emulating the relevant steps in the proof of [Kauta 2012, Theorem]. To achieve this, it suffices to show that $O_{l}\left(J\left(A_{f}\right)\right)=\sum_{\tau \in G} S k_{\tau} x_{\tau}$ for some $k_{\tau} \in K^{\#}$, and that $I_{\tau} f\left(\tau, \tau^{-1}\right)=J(V) S$ for each $\tau \in G$, where $I_{\tau}$ is as in Lemma 2.4. The second assertion follows from Lemma 2.4(3). As for the first one, we first note that $O_{l}\left(J\left(A_{f}\right)\right)$ is a $V$-order in $\Sigma_{f}$, by [Kauta 1997a, Corollary 1.3]. By Lemma 2.5, $O_{l}\left(J\left(A_{f}\right)\right)=\sum_{\tau \in G} S k_{\tau} x_{\tau}$ for some $k_{\tau} \in K^{\#}$ if and only if it is finitely generated over $V$.

Since for all $\tau, \gamma \in G$ and every maximal ideal $M$ of $S$ we have $f(\tau, \gamma) \notin M^{2}$, we conclude from Lemma 2.3 that $f(G \times G) \subseteq U(R)$, hence $B_{f}$ is Azumaya over $W$. Therefore $J\left(B_{f}\right)=J(W) B_{f}=J(W)\left(W A_{f}\right)=J(W) A_{f} \subseteq J\left(A_{f}\right)$, and $A_{f} / J\left(B_{f}\right)$ is a $\tilde{V}$-order in $B_{f} / J\left(B_{f}\right)$. Since $O_{l}\left(J\left(A_{f}\right)\right)$ is a $V$-order containing $A_{f}, O_{l}\left(J\left(A_{f}\right)\right) W$ is a $W$-order containing $B_{f}$, so $O_{l}\left(J\left(A_{f}\right)\right) W=B_{f}$, since $B_{f}$ is a maximal $W$-order in $\Sigma_{f}$, and hence $O_{l}\left(J\left(A_{f}\right)\right) \subseteq B_{f}$. Therefore $O_{l}\left(J\left(A_{f}\right)\right) / J\left(B_{f}\right)$ is a $\tilde{V}$-order in $B_{f} / J\left(B_{f}\right)$, a central simple $\bar{W}$-algebra. Since $\tilde{V}$ is a DVR of $\bar{W}, O_{l}\left(J\left(A_{f}\right)\right) / J\left(B_{f}\right)$ must be finitely generated over $\tilde{V}$, by [Reiner 2003, Theorem 10.3], hence there exists $a_{1}, a_{2}, \ldots, a_{n} \in O_{l}\left(J\left(A_{f}\right)\right)$ such that $O_{l}\left(J\left(A_{f}\right)\right)=a_{1} V+a_{2} V+\cdots+a_{n} V+J\left(B_{f}\right)=a_{1} V+a_{2} V+\cdots+a_{n} V+A_{f}$, a finitely generated $V$-module. Thus $O_{l}\left(J\left(A_{f}\right)\right)=A_{f}$.

As in the proof of [Morandi 1992, Lemma 4.11], we have $O_{l}\left(J\left(A_{f} / J\left(B_{f}\right)\right)\right)=$ $O_{l}\left(J\left(A_{f}\right) / J\left(B_{f}\right)\right)=O_{l}\left(J\left(A_{f}\right)\right) / J\left(B_{f}\right)=A_{f} / J\left(B_{f}\right)$, where $O_{l}\left(J\left(A_{f} / J\left(B_{f}\right)\right)\right)$ 
and $O_{l}\left(J\left(A_{f}\right) / J\left(B_{f}\right)\right)$ are defined accordingly. Since $\tilde{V}$ is a DVR of $\bar{W}, A_{f} / J\left(B_{f}\right)$ is a hereditary $\tilde{V}$-order in the central simple $\bar{W}$-algebra $B_{f} / J\left(B_{f}\right)$, hence $A_{f}$ is semihereditary by [Morandi 1992, Lemma 4.11].

We summarize these results as follows.

Theorem 3.4. Given a crossed-product order $A_{f}$ :

(1) It is semihereditary if and only if for all $\tau, \gamma \in G$ and every maximal ideal $M$ of $S, f(\tau, \gamma) \notin M^{2}$; if and only if for each $\gamma \in G$ and each maximal ideal $M$ of $S, f\left(\tau, \tau^{-1}\right) \notin M^{2}$.

(2) If $J(V)$ is a nonprincipal ideal of $V$, then $A_{f}$ is semihereditary if and only if it is Azumaya over $V$, if and only if $H=G$.

We now lump together several corollaries of the theorem above, generalizing results in [Kauta 2012].

Corollary 3.5. (1) Given a crossed-product order $A_{f}$ :

(a) It is a valuation ring if and only if given any maximal ideal $M$ of $S$, $f\left(\tau, \tau^{-1}\right) \notin M^{2}$ for each $\tau \in G$, and there exists a set of right coset representatives $g_{1}, g_{2}, \ldots, g_{r}$ of $D_{M}$ in $G$ (that is, $G$ is the disjoint union $\left.\bigcup_{i} D_{M} g_{i}\right)$ such that for all $i, f\left(g_{i}, g_{i}^{-1}\right) \notin M$.

(b) If $V$ is indecomposed in $K$, then it is a valuation ring if and only if for each $\tau \in G, f\left(\tau, \tau^{-1}\right) \notin J(S)^{2}$.

(2) Suppose the crossed-product order $A_{f}$ is primary. Then it is a valuation ring if and only if there exists a maximal ideal $M$ of $S$ such that for each $\tau \in D_{M}$, $f\left(\tau, \tau^{-1}\right) \notin M^{2}$.

(3) Suppose the crossed-product order $A_{f}$ is semihereditary. Then $A_{f_{L, U}}$ is a semihereditary order in $\Sigma_{f_{L, U}}$ for each intermediate field $L$ of $F$ and $K$, and every valuation ring $U$ of $L$ lying over $V$.

(4) Suppose the crossed-product order $A_{f}$ is semihereditary. Then $A_{f_{M}}$ is a valuation ring of $\Sigma_{f_{M}}$ for each maximal ideal $M$ of $S$.

We end by observing yet another peculiarity of these crossed-product orders. The proposition below not only strengthens Lemma 2.1(2) when the $V$-order $A$ is taken to be the crossed-product order $A_{f}$, but also generalizes [Haile and Morandi 1993, Proposition 2.10] to the case where $V$ is not necessarily indecomposed in $K$.

Proposition 3.6. Suppose the crossed-product order $A_{f}$ is extremal and $W$ is a valuation ring of $F$ with $V \varsubsetneqq W$. Then $W A_{f}$ is Azumaya over $W$.

Proof. This follows from Lemma 2.2(1), Theorem 3.4(1), Lemma 2.3, and Proposition 3.1. 


\section{References}

[Dubrovin 1982] N. I. Dubrovin, "Noncommutative valuation rings", Trudy Moskov. Mat. Obshch. 45 (1982), 265-280. In Russian; translated in Trans. Moskow Math. Soc. 45 (1984), 273-287. MR 85d:16002 Zbl 0543.16010

[Dubrovin 1984] N. I. Dubrovin, "Noncommutative valuation rings in simple finite-dimensional algebras over a field", Mat. Sb. (N.S.) 123(165):4 (1984), 496-509. In Russian; translated in Math. USSR-Sb. 51:2 (1985), 493-505. MR 85j:16020 Zbl 0573.16012

[Endler 1972] O. Endler, Valuation theory, Springer, New York, 1972. MR 50 \#9847 Zbl 0257.12111

[Haile 1987] D. E. Haile, "Crossed-products orders over discrete valuation rings", J. Algebra 105:1 (1987), 116-148. MR 88b:16013 Zbl 0609.16004

[Haile and Morandi 1993] D. E. Haile and P. J. Morandi, "On Dubrovin valuation rings in crossed product algebras”, Trans. Amer. Math. Soc. 338:2 (1993), 723-751. MR 93j:16010 Zbl 0798.16031

[Haile et al. 1983] D. E. Haile, R. G. Larson, and M. E. Sweedler, "A new invariant for $\mathbb{C}$ over $\mathbb{R}$ : almost invertible cohomology theory and the classification of idempotent cohomology classes and algebras by partially ordered sets with a Galois group action", Amer. J. Math. 105:3 (1983), 689-814. MR 84k:12014 Zbl 0523.13005

[Haile et al. 1995] D. E. Haile, P. J. Morandi, and A. R. Wadsworth, "Bézout orders and Henselization”, J. Algebra 173:2 (1995), 394-423. MR 96i:16025 Zbl 0826.16010

[Kauta 1997a] J. S. Kauta, "Integral semihereditary orders, extremality, and Henselization", J. Algebra 189:2 (1997), 226-252. MR 98d:16030 Zbl 0871.16009

[Kauta 1997b] J. S. Kauta, "Integral semihereditary orders inside a Bézout maximal order", J. Algebra 189:2 (1997), 253-272. MR 98d:16031 Zbl 0871.16010

[Kauta 2001] J. S. Kauta, "Crossed product orders over valuation rings", Bull. London Math. Soc. 33:5 (2001), 520-526. MR 2002d:16023 Zbl 1029.16012

[Kauta 2012] J. S. Kauta, "On a class of hereditary crossed-product orders", 2012. To appear in Proc. Amer. Math. Soc. arXiv 1210.1065

[Morandi 1992] P. J. Morandi, "Maximal orders over valuation rings", J. Algebra 152:2 (1992), 313-341. MR 93k:16028 Zbl 0781.16007

[Reiner 2003] I. Reiner, Maximal orders, London Mathematical Society Monographs. New Series 28, Clarendon, Oxford, 2003. MR 2004c:16026 Zbl 1024.16008

Received October 5, 2011. Revised March 27, 2012.

JOHN S. KAUTA

DEPARTMENT OF MATHEMATICS

FACULTy OF SCIENCE

UNIVERSITI BRUNEI DARUSSALAM

BANDAR SERI BEGAWAN BE1410

BRUNEI DARUSSALAM

john.kauta@ubd.edu.bn 


\title{
PACIFIC JOURNAL OF MATHEMATICS
}

\author{
http://pacificmath.org \\ Founded in 1951 by \\ E. F. Beckenbach (1906-1982) and F. Wolf (1904-1989)
}

\section{EDITORS}

V. S. Varadarajan (Managing Editor)

Department of Mathematics

University of California

Los Angeles, CA 90095-1555

pacific@math.ucla.edu

Vyjayanthi Chari

Department of Mathematics

University of California

Riverside, CA 92521-0135

chari@math.ucr.edu

\section{Robert Finn}

Department of Mathematics Stanford University

Stanford, CA 94305-2125

finn@math.stanford.edu

Kefeng Liu

Department of Mathematics

University of California

Los Angeles, CA 90095-1555

liu@math.ucla.edu
Darren Long

Department of Mathematics

University of California

Santa Barbara, CA 93106-3080

long@math.ucsb.edu

Jiang-Hua Lu

Department of Mathematics

The University of Hong Kong

Pokfulam Rd., Hong Kong jhlu@maths.hku.hk

Alexander Merkurjev

Department of Mathematics

University of California

Los Angeles, CA 90095-1555

merkurev@math.ucla.edu
Sorin Popa

Department of Mathematics University of California

Los Angeles, CA 90095-1555 popa@math.ucla.edu

Jie Qing

Department of Mathematics

University of California

Santa Cruz, CA 95064

qing@cats.ucsc.edu

Jonathan Rogawski

Department of Mathematics

University of California

Los Angeles, CA 90095-1555

jonr@math.ucla.edu

\section{PRODUCTION}

pacific@math.berkeley.edu

\section{SUPPORTING INSTITUTIONS}

ACADEMIA SINICA, TAIPEI

CALIFORNIA INST. OF TECHNOLOGY INST. DE MATEMÁTICA PURA E APLICADA KEIO UNIVERSITY

MATH. SCIENCES RESEARCH INSTITUTE NEW MEXICO STATE UNIV.

OREGON STATE UNIV.

\author{
STANFORD UNIVERSITY \\ UNIV. OF BRITISH COLUMBIA \\ UNIV. OF CALIFORNIA, BERKELEY \\ UNIV. OF CALIFORNIA, DAVIS \\ UNIV. OF CALIFORNIA, LOS ANGELES \\ UNIV. OF CALIFORNIA, RIVERSIDE \\ UNIV. OF CALIFORNIA, SAN DIEGO \\ UNIV. OF CALIF., SANTA BARBARA
}

\author{
UNIV. OF CALIF., SANTA CRUZ \\ UNIV. OF MONTANA \\ UNIV. OF OREGON \\ UNIV. OF SOUTHERN CALIFORNIA \\ UNIV. OF UTAH \\ UNIV. OF WASHINGTON \\ WASHINGTON STATE UNIVERSITY
}

These supporting institutions contribute to the cost of publication of this Journal, but they are not owners or publishers and have no responsibility for its contents or policies.

See inside back cover or pacificmath.org for submission instructions.

The subscription price for 2012 is US \$420/year for the electronic version, and \$485/year for print and electronic.

Subscriptions, requests for back issues from the last three years and changes of subscribers address should be sent to Pacific Journal of Mathematics, P.O. Box 4163, Berkeley, CA 94704-0163, U.S.A. Prior back issues are obtainable from Periodicals Service Company, 11 Main Street, Germantown, NY 12526-5635. The Pacific Journal of Mathematics is indexed by Mathematical Reviews, Zentralblatt MATH, PASCAL CNRS Index, Referativnyi Zhurnal, Current Mathematical Publications and the Science Citation Index.

The Pacific Journal of Mathematics (ISSN 0030-8730) at the University of California, c/o Department of Mathematics, 969 Evans Hall, Berkeley, CA 94720-3840, is published monthly except July and August. Periodical rate postage paid at Berkeley, CA 94704, and additional mailing offices. POSTMASTER: send address changes to Pacific Journal of Mathematics, P.O. Box 4163, Berkeley, CA 94704-0163.

PJM peer review and production are managed by EditFLOW ${ }^{\mathrm{TM}}$ from Mathematical Sciences Publishers.

PUBLISHED BY PACIFIC JOURNAL OF MATHEMATICS

at the University of California, Berkeley 94720-3840

A NON-PROFIT CORPORATION

Typeset in LATEX

Copyright $(02012$ by Pacific Journal of Mathematics 


\section{PACIFIC JOURNAL OF MATHEMATICS}

Volume $259 \quad$ No. $2 \quad$ October 2012

Flag subdivisions and $\gamma$-vectors

257

Christos A. ATHANASIAdis

Rays and souls in von Mangoldt planes

279

IGOR BELEGRADEK, ERIC CHOI and NOBUHIRO INNAMI

Isoperimetric surfaces with boundary, II

ABRAHAM FRANDSEN, DONALD SAMPSON and NEIL

STEINBURG

Cyclic branched coverings of knots and quandle homology

YUICHI KABAYA

On a class of semihereditary crossed-product orders

JOHN S. KAUTA

An explicit formula for spherical curves with constant torsion

DEMETRE KAZARAS and IVAN STERLING

Comparing seminorms on homology

JEAN-FranÇOIS LAFONT and CHRISTOPHE PITTET

Relatively maximum volume rigidity in Alexandrov geometry

NAN LI and XIAOCHUN RONG

Properness, Cauchy indivisibility and the Weil completion of a group of 421 isometries

Antonios Manoussos and Polychronis Strantzalos

Theta lifts of strongly positive discrete series: the case of $(\tilde{\mathrm{Sp}}(n), O(V)) 445$

IVAN MATIĆ

Tunnel one, fibered links

MATt RATHBUN

Fusion symmetric spaces and subfactors

HANS WENZL 